\title{
Turning Need into Demand for Wi-Fi Broadband Internet Access on Trains
}

\author{
Sajjad Karim \\ Dept of Telecom Engineering \\ Foundation University \\ Institute of Engineering and \\ Management Sciences, \\ Pakistan
}

\author{
Shoaib Khan
RAN Optimizer at Telefonica
O2, United Kingdom \\ Shoaib Khan
RAN Optimizer at Telefonica
O2, United Kingdom \\ Shoaib Khan
RAN Optimizer at Telefonica
O2, United Kingdom
}

Ahmed Ali Qureshi

Dept of Software Engineering

Foundation University

Institute of Engineering and

Management Sciences,

Pakistan

\author{
Imran Daud \\ Dept of Telecom Engineering \\ Foundation University \\ Institute of Engineering and \\ Management Sciences, \\ Pakistan
}

\begin{abstract}
Nowadays, it is the demand of every internet user to stay connected most of the time even if the user is on the move. The core focus of this paper is on internet connectivity with moving vehicles. Wireless internet access on trains is not a standardized technology yet, therefore, it has diverse and vendor specific architecture. The authors have presented the physical architecture of internet access on moving vehicles, specifically trains and the logical architecture to give a clear idea that how the service will work at each layer of TCP/IP protocol stack. In order to observe the data rates offered on board, and experience Wi-Fi internet access onboard, the authors chose Great North Eastern Railway (GNER) route from London Kings Cross station till Peterborough (UK). For the network data rate analysis the authors chose Bandwidth Monitor a freeware used to monitor network and Internet bandwidth. The authors observed the data rates on GNER Wi-Fi internet on basis of three different scenarios i.e., Basic Browsing, Live Radio(Voice),VOIP services and video streaming Consequently, suggestions for improving quality of experience and market penetrations plans to make this technology successful in terms of generating revenues for the operators are given.
\end{abstract}

\section{Index Terms}

Wi Fi, Internet access, Trains, broad band, Thin client based architecture.

\section{INTRODUCTION}

Evolution in the technology has taken the humanity beyond imaginations in the last few decades. The most evident and impressive innovations are in the field of computer science and telecommunications. Most of the technological equipment under use went through certain basic evolutionary changes. The size of the devices underuse is reduced significantly, "Miniaturization". The devices have become easier to use and handle, "Ergonomics". The wired connectivity between the devices is minimal, "Wireless". The data transfer among or between the devices is getting faster each day, "Data Rates". A single equipment or device provides the various functionalities, "Multifunctional devices". Amongst all other advances in the technology internet is one of the most successful. In the present age internet has become the part of life for every human beings. Every business organization and the home user and even a mobile phone user connects to the internet for the purposes such as checking mail, browsing internet, community messaging, office work, stock brokerage-banking, study purposes, looking for cheaper flights, train tickets downloading the content of interest and others. It is the demand and necessity of every internet user to stay connected most of the time. The demand was to provide mobility to the internet users, so that even if the internet user is on the move he can still benefit from the connectivity and data transfers. To complement the demand the concept of wireless internet connectivity was set forth. There are various technologies and standards that provide wireless Internet access to the mobile users. Most prominent standards are shown in Table-1.1.

As the technology kept on evolving, the efforts and research work was then dedicated towards the wireless internet access on the moving vehicles such as cars, buses, trains and aircrafts. The core focus of this research is on internet connectivity in moving vehicles. There can be a number of scenarios in which a commuter on train can benefit from the internet access on moving train. Some of the scenarios are discussed below to get a clear idea.

Table 1.1 Wireless Technologies that can provide wireless Internet Access [1]

\begin{tabular}{|c|c|c|}
\hline CATEGORY & TECHNOLOGY & EEEE-Standard \\
\hline \multirow{3}{*}{ WPAN } & Bluetooth & 802.15 .1 \\
\hline & Zigbee & 802.15 .4 \\
\hline & UWB & 802.15 \\
\hline \multirow[t]{2}{*}{ WLAN } & WiFi & 802.11 \\
\hline & WLMax & 802.16 \\
\hline \multirow[t]{3}{*}{ WMLAN } & Iburst & 802.20 \\
\hline & PHS & $802.1 \mathrm{X}$ \\
\hline & GPRS & $2.5 \mathrm{G}$ Celludar \\
\hline \multirow[t]{3}{*}{ WWAN } & WCDMA & 3G Cellualar \\
\hline & CDMA-2000 & $3 \mathrm{G}$ Cellualar \\
\hline & TD-DCMA & 3G Cellualar \\
\hline
\end{tabular}

\subsection{On board Wireless Connectivity Scenarios}

1.1.1 An employee, who is a daily commuter of a train from his home to office. It takes an hour's time to reach his office via train. The best situation is that the train service also offers internet access while on the move. The employee can make use 
of this service to start his office work rite at the moment when he grabs his seat in the train. Setting up his appointments and replying to the official mails.

1.1.2 A student commuting on train in a journey towards his university can check his student mail, class schedule and can complete his unfinished assignment on his journey.

1.1.3 A stockbroker can be updated all the time with the ups and downs in the stock rates during his journey. He can bid for a new share or sell his current share without suffering from any delays.

1.1.4 A bored commuter who has to undergo a long journey can watch a movie or listen to some good quality music during his journey. In case he likes to read books he can go to a web page where he can find some useful material.

1.1.5 A tourist can find the nearest taxi stand, and places to visit near a particular station that he intends to visit. There can be a never-ending list of scenarios, which clearly highlights the significance of Internet access while on the move.

\subsection{Key concerns towards Quality of Experience (QoE)}

In the best case if the service is provided with the best available technology availability and data rates all the commuters will be satisfied and the technology will be successful. But unfortunately providing internet access on a high speed train is very difficult. It is difficult to provide consistent and satisfactory services. Further the authors have discussed architecture, technological aspects and best practices in detail. The key concerns towards the QoE are as follows.

1. Is the commuter getting the data rates at which his communication is meaningful?

2. Are there any no-service areas or disconnect points during the journey?

3. Is the commuter satisfied with the service provided?

4. Is there any room for enhancement in data rate and availability?

5. Is the communication on wireless link secure?

6. Is the information provided on the web pages is current and updated?

7. Does the surfing offer minimal delays so that the commuter is not annoyed?

8. What is the pricing for the service? Is the commuter satisfied with the service and is willing to pay for it?
9. What is the extra value added in service, to make the service better as compared to other onboard internet providers?

There are many Train services across the world that is providing data services on the move, not only the trains but also some shipping companies and road-travelling companies are also providing Internet services on the move as well. Some of the leading examples are Virgin Trains cover long distance railway services in U.K with Wi-Fi internet access, QinetiQ has derived full trails of Wi-Fi internet access of five major train routes across Europe. But still this technology has not become a breakthrough in the industry yet. The authors have focused on the reasons and strategies towards successful solutions later in the paper.

\section{DIVERISTY IN PHYSICAL ARCHITECTURE}

Wireless internet access on trains is not a standardized technology yet, therefore, it has diverse and vendor specific architecture. The architectural components and technology is entirely based on service provider or service operator's choice. However, the basic architectural components and technological solutions available are discussed in detail. The vendor specific solutions and protocol specific details will be discussed later. To understand the overall architecture following two basic wireless environments, working coherently to provide a single service, were kept in focus.

1. A Wi-Fi onboard connectivity. In which each wireless terminal user is connected to the onboard access point directly using his Wi-Fi $802.11 \mathrm{a} / \mathrm{b} / \mathrm{g}$ enabled equipment, it may be a laptop or PDA.

2. A hybrid or technology specific antenna mounted on the train roof to achieve wireless internet connectivity through some technological solution. The on-roof antenna may connect through WiMAX, GPRS, satellite or broadband power line.

The passenger sitting on train is equipped with a Terminal Equipment (TE) that might be a Wi-Fi enabled laptop or PDA. The TE has a wireless connection with the Access Point (AP) on train. The AP is connected to the Rail Point server (RPS). The authentication and authorization checks for the passenger are done at the RPS

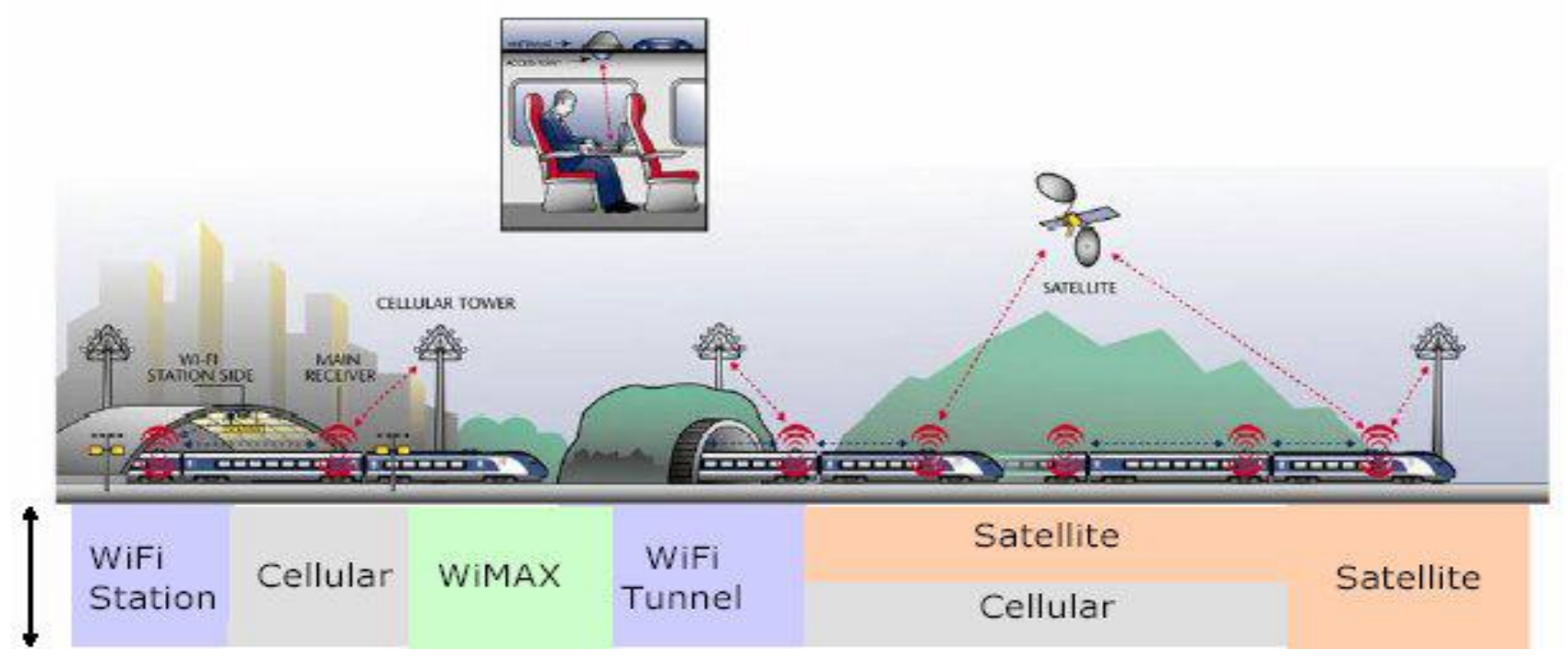

Figure 2.0 External Wireless Link Diversity for train roof antenna 
The RPS on train connects to the Rail Point Gateway RPG on the ground employing one of the wireless wide area network links such as Wi-Fi hotspot links, cellular links, GPRS, CDMA, WCDMA, WiMAX broadband access, 2-way, 1-way satellite link, bi-directional antenna arrays or broadband on power lines as shown graphically in Figure-2.0.

The decision for the specific technology of RPS to RPG connection can be made on following points.

1. The cost of deployment of a particular technology, CAPEX.

2. The cost of link establishments and operating cost OPEX.

3. Requirement of minimum data rate of the link, bandwidth requirements.

4. Requirement of availability of service in urban as well as rural areas.

5. Quality of service and quality of experience requirements.

Each technological solution has its strengths and weaknesses depending on the scenario; thus, every technology can be beneficial. But none of them can be called an ultimate solution. The authors have established technical details for each of the available choices for rail to ground link.

\subsection{Wi-Fi Based Connectivity}

Wi-Fi stands for Wireless Fidelity, it is IEEE 802.11 standard for wireless connectivity between devices. It operates on 2.4 $\mathrm{GHz}$ and $5 \mathrm{GHz}$ ISM band. Most popular and commercial Wi-Fi standards are $802.11 \mathrm{a} / \mathrm{b} / \mathrm{g}$. Currently used commercial products are based on 802.1g standard that is backwards compatible. The max range of $\mathrm{Wi}-\mathrm{Fi}$ based link is 50 meters indoor and 150 meters outdoor. The extended wireless link may range up to 300

meters. But Wi-Fi based link offers data rates of 11 to 54 Mbps. Since the Wi-Fi based solution offers very low mobility it cannot be accepted as a solution for out of train connectivity. But onboard this is the best solution, while the passenger is inside the train. And the same technology can be used for RPS to RPG connectivity while the train is inside the station or at platform. This

way the authors will also be able to make most of the stations as Wi-Fi hotspots. Similarly, while the train is passing through a tunnel or a bridged area where there is no other service possible. For example the tunnel between Malmo Sweden and Copenhagen Denmark is almost 18 miles long and 12 miles under water. In this situation Wi-Fi based access may be a useful solution.

\subsection{WiMAX based Connectivity}

WiMAX stands for Worldwide Interoperability for Microwave Access. It is based on IEEE 802.16 standard for interoperability. WiMAX can be considered as a long-range connectivity for broadband services like telephony, data services, streaming media broadcast etc. WiMAX provides Metropolitan Area Network (MAN) connectivity. WiMAX operates at 2 to $66 \mathrm{GHz}$ frequency range. This is a very high frequency and low bandwidth spectrum, and more susceptible to noise. WiMAX offers data rates up to $72 \mathrm{Mbps}$ and provides coverage up to 6 miles or 10 kilometers. WiMAX is said to be the future of Broadband Wireless Access (BWA) technology. There are many other standards similar to WiMAX for long range MAN broadband access like HIPERMAN, WiBro and iBurst. Although WiMAX seems to be a better solution since it offers high data rates along with a considerable range as depicted in Figure-2.2. But the problem is the cost of deployment of WiMAX radio base stations along hundreds of miles of railroad tracks. The core advantage using WiMAX is its data rate, 72 Mbps data rate is a very high and desired by every operator. Caltrain is the name of train service in USA working on the railway track of 16 miles between San Francisco and San Jose. Caltrain provides internet access inside the train using highspeed broadband access technology, WiMAX that provides continuous high speed Internet access along a rail line at travel speeds of up to 79 miles per hour [2]

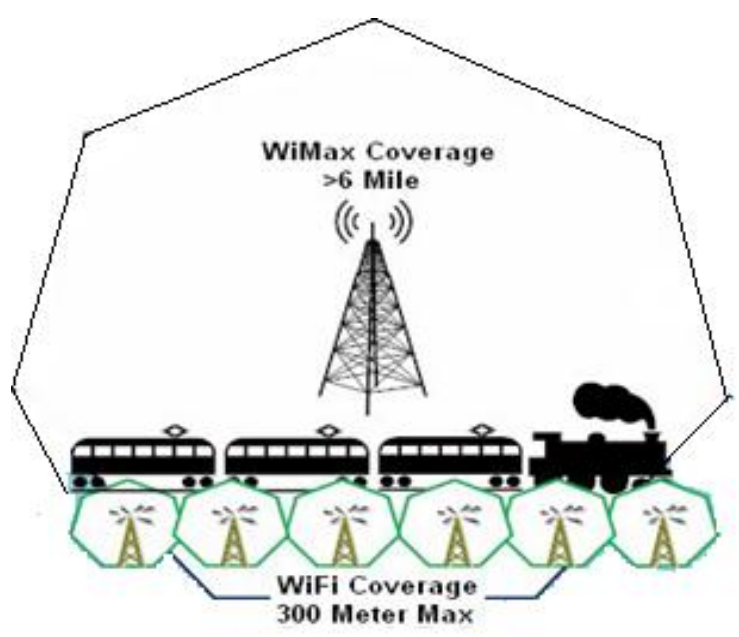

\section{Figure 2.2 Visual difference between Wi-Fi and WiMAX coverage area}

\subsection{Terrestrial Cellular based Connectivity}

Cellular based mobile technologies are the oldest commercial technologies that provide wireless data access to the devices. Cellular based connectivity can be based on either of the technologies like GPRS, EDGE, CDMA, WCDMA, TDDCDMA, and UMTS. The details of data rates offered and maximum ranges are given in the Table-2.3.

The table clearly shows the strengths and weaknesses of cellular terrestrial internet providers. The data rates provided by all the above mentioned technologies have comparatively low services like file transfers, streaming media and fast browsing is not possible at such data rates. On the other hand, the range of cellular transmitters is way higher as compared to WiMAX and Wi-Fi technologies. The overall cost of deployments and operating cost is also much lower as compare to WiMAX and Wi-Fi. The cellular based internet connectivity may be provided by the train service operator or directly by the mobile network company. The passenger only needs a GPRS, or CDMA enabled phone to connect to his laptop as a wireless modem.

\subsection{Use of Directional Antenna Arrays}

Satellite based connectivity for broadband service on train can prove to be a better solution since satellites provide high range coverage as compared to WiMAX and cellular technologies and lower handovers required for mobility management. Satellite based coverage is much more robust towards high speeds, as the high-speed trains might accelerate up to $300 \mathrm{Mph}$. Most of the requirements and depiction of satellite-based connectivity to train is shown in Figure-2.5 below. 


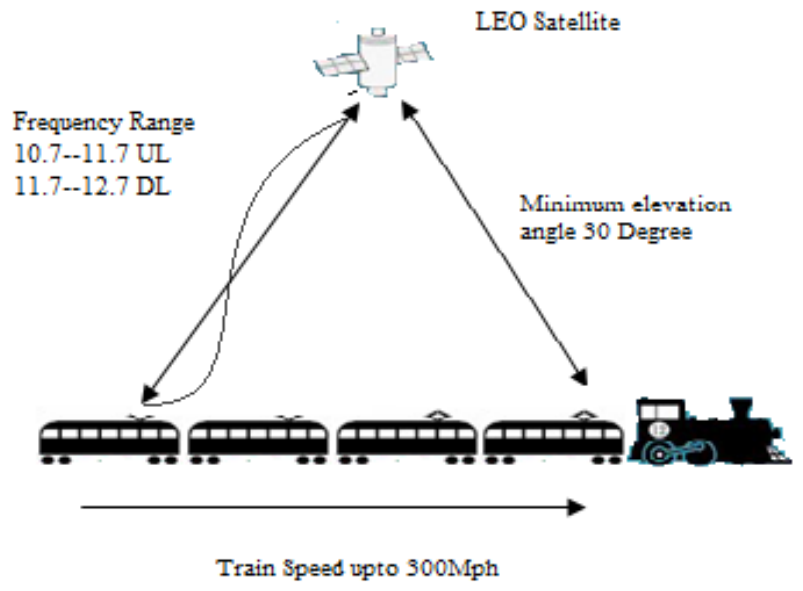

Figure 2.5 Satellite to train connectivity

\subsection{Key Problems faced in Satellite-based connectivity}

Key problems faced in satellite-based connectivity are: Need for satellite to be in Line of Sight (LoS), automatic tracking of satellite transceivers, mechanical requirements for directional antennas and others. Cost of uploading, downloading in bits per second, high power requirements on the train roof transceiver and interference problems.
Most of the commercial train services today are using satellitebased connection only for forward channel and use WiMAX or cellular terrestrial connections for return channel. In case on No-Line of Sight (NLoS), train services completely rely on cellular technologies or WiMAX.

\subsection{Optic Fiber Access Network (OFAN) Based Connectivity}

OFAN based internet connectivity to fast moving trains is another promising and worth mentioning solution. This solution is based on a vision to provide high bandwidth applications to the daily commuters on trains. It is not simply an idea of mounting directional antennas along the railway trackside and giving each of them back-end connectivity through optic fibers. The OFAN-Radio connectivity is based on RoF technology. RoF stands radio through optic fiber. It is a system of fiber-fed distributed antenna network. The key feature of RoF is to transfer all the processing functionalities related to signal processing, modulation and demodulation to a central control unit. And all base stations are only used for radio transmission and reception such base stations are known as RAUs Remote Antenna Units. As a result a very low capital expenditure is required and better controlling facility.

Table 2.3 Data Rate, Range and operating frequency of terrestrial cellular Internet service providers

\begin{tabular}{|c|c|c|c|c|}
\hline Technology & $\begin{array}{l}\text { Data Rate } \\
\text { Downlink }\end{array}$ & $\begin{array}{c}\text { Data Rate } \\
\text { Uplink }\end{array}$ & $\begin{array}{c}\text { Maximum } \\
\text { Range }\end{array}$ & Operating frequency band \\
\hline CSD & $9.6 \mathrm{Kbps}$ & $9.6 \mathrm{Kbps}$ & $15-30 \mathrm{Km}$ & $450,900,1800 \mathrm{Mhz}$ \\
\hline HSCSD & $28.8 \mathrm{Kbps}$ & $14.4 \mathrm{Kbps}$ & $15-30 \mathrm{Km}$ & $450,900,1800 \mathrm{Mhz}$ \\
\hline HSCSD & $43.2 \mathrm{Kbps}$ & $14.4 \mathrm{Kbps}$ & $15-30 \mathrm{Km}$ & $450,900,1800 \mathrm{Mhz}$ \\
\hline GPRS & $80.0 \mathrm{Kbps}$ & $\begin{array}{c}\text { 20.0Kbps Class } \\
8,10, \mathrm{CS}-4\end{array}$ & $15-30 \mathrm{Km}$ & $450,900,1800 \mathrm{Mhz}$ \\
\hline GPRS & $60.0 \mathrm{Kbps}$ & $\begin{array}{c}40.0 \mathrm{Kbps} \text { Class } \\
10, \mathrm{CS}-4\end{array}$ & $15-30 \mathrm{Km}$ & $450,900,1800 \mathrm{Mhz}$ \\
\hline $\begin{array}{l}\text { CDMA } 2000 \\
\text { 1xRTT }\end{array}$ & $307 \mathrm{Kbps}$ & $153 \mathrm{Kbps}$ & $15-30 \mathrm{Km}$ & $450,900,1800 \mathrm{Mhz}$ \\
\hline $\begin{array}{c}\text { CDMA } 2000 \\
1 \times E V\end{array}$ & $2.4 \mathrm{Mbps}$ & $153 \mathrm{Kbps}$ & $15-30 \mathrm{Km}$ & $450,900,1800 \mathrm{Mhz}$ \\
\hline WCDMA & $\begin{array}{l}\text { 2.3Mbps Physical Link } \\
\text { 384Kbps offered rate }\end{array}$ & $\begin{array}{l}\text { 2.3Mbps Physical Link } \\
\text { 384Kbps offered rate }\end{array}$ & $15-30 \mathrm{Km}$ & $1800-2200 \mathrm{Mhz}$ \\
\hline UMTS & $\begin{array}{l}\text { 2Mbps Physical Link } \\
384 \mathrm{Kbps} \text { offered rate }\end{array}$ & $\begin{array}{l}\text { 2Mbps Physical Link } \\
384 \mathrm{Kbps} \text { offered rate }\end{array}$ & $15-30 \mathrm{Km}$ & $1800-2200 \mathrm{Mhz}$ \\
\hline
\end{tabular}

The only constraint in employing RoF technology is mounting of a huge number of RAUs along the trackside. Contrary to initial cost it provides a high bandwidth and real-time solution to passengers like video streaming and voice services [3].

\subsection{Broadband through Power Lines based Connectivity}

Power line communication or broadband over power line is a new idea set forth by Federal Communications Commission (FCC). It is the concept of sending voice or data at lower frequency RF signal over physical lines that are used for electrical power supply. This is an emerging technology and is tested at very few places as yet. Employing BPL technology 
the data rates can be achieved for $500 \mathrm{Kbps}$ to $3 \mathrm{Mbps}$. BPL has already been tested in some countries for broadband services of homes and static users. Different researches are trying to employee BPL technology over power lines to trains but commercial products are yet to be tested or seen.

\subsection{Scenario based Hybrid Connectivity}

Six different architectural models to connect train to terrestrial internet services are discussed, but in every scenario there were certain strengths and weaknesses. On the basis of above discussion one can conclude following Key Performance Indicators (KPIs). Towards quality of experience that can be provided in Internet services on trains.

- Data rate on uplink and downlink.

- Roundtrip Time (RTT) for a signal

- Availability of service.

- Handover success/failure rate.

The best approach followed by almost all the internet service providing railroad services is the use of hybrid antennas. A hybrid antenna has a capability of seamless

vertical handovers between various technologies. The decision can be made on following parameters.

1. Rx-level, received signal levels on the basis of signal strength.

2. Rx-Qual, received signal quality on the basis of Bit Error Rate (BER).

3. Network availability.

4. Priority based scheduling.

5. Cost based scheduling.

6. Minimum data rate threshold.

There might be two or more technologies like satellite based connectivity and cellular terrestrial based connectivity available at the same time but it depends on the priority and parameter settings which technology the antenna will camp with. Now the core problem is to design an antenna that supports vertical handovers with optimal performance and minimum interference towards hybrid technologies. Fast moving trains for intercity travelling pass through various locations and geographical terrains, while travelling through urban, sub-urban and rural areas. In the way these trains have to pass through various tunnels and bridges. At each location there might be certain technology providing better connectivity than the other. It depends on the smartness of antenna designs that how fast and accurate handovers it makes between technologies or within the same technology, with minimum delays and no data losses, seamless to the internet users or passengers in the train. There are two basic types of handovers.

1. Vertical or Inter Segment Handovers.

2. Horizontal or Intra Segment Handovers.

For a successful handover execution various aspects need to be addressed properly such as graceful transfer of control and data, bearer setup and release, switching of control, new route allocation for data, graceful release of previous connection. During handover execution QOS must be maintained and it should be seamless to the Internet users inside the train. In horizontal handovers within the same technology handover occurs at data link layer DLL, whereas in vertical handover or ISHO it occurs at network layer. The key solution for handovers outside the train environment is to make use of hybrid antenna diversity at terminal internetworking unit $\mathrm{T}$ -
IWU. Antenna diversity requires two sets of antennas to be mounted on the train roof. Normally hybrid antennas have built in diversity. Using this technique whenever handover margin or threshold is met handover decision is made at the terminal end and handover execution takes place. In case of handover decisions it is always terminal assisted handover not a network-assisted handover. One of the practical scenarios is when a train enters a tunnel while previously it is using a satellite or WiMAX connection. Instantaneously the handover decision for Wi-Fi based connectivity has to be made inside the tunnel seamless to the passenger using internet inside the train. [4]

\subsubsection{Huber and Suhner Antennas}

Huber and Suhner is one of the leading companies manufacturing antennas for hybrid technology providing vertical handover facility between various technologies. The commercial antenna models in market are.

- $\quad$ SWA $0859 / 360 / 4 / 0 / \mathrm{V}$.

- $\quad$ SWA 0859/360/4/0/DF.

- $\quad$ SWA 0859/360/4/0/DFRX30

These antennas provide support for GSM-GPRS, GSMEDGE, GSM-PCS, UMTS, WLAN, Wi-Fi $802.11 \mathrm{a} / \mathrm{b} / \mathrm{g}$ and WiMAX technology vertical ISHO handovers. Apart from wide range of technology they can be utilized for any technology between $870 \mathrm{MHz}$ to $5.9 \mathrm{GHz}$ [5].

\section{LOGICAL ARCHITECTURAL MODELS}

The authors have discussed in detail the physical architecture of internet access on moving vehicles, specifically trains. The prior discussion only gives detail of the physical layer, now the focus will be on the logical architecture so that one has a clearer idea that how the service will work at each layer of TCP/IP protocol stack. Providing internet access on moving vehicles is a purely multi-domain and multi-homed approach. The key concept is to make it a multi-vendor and unified standard approach as well. Priory stated approaches like WiMAX, Wi-Fi or cellular based internet connectivity based approach provide good data rates at high speed moving trains up to $150 \mathrm{Mph}$ or more. But the key problem arises while providing inter standard handovers and mobility support. The aggregation of data traffic from fast moving vehicles to service providers requires fast switching between protocols and technologies seamless to the end user. The configuration needs to be in time. To provide a fast switching various aggregation techniques and models are set forth. These models and architecture have core focus on the perceived quality of service QoS by the end user.

\subsection{BIT based Approach}

Broadband wireless internet access on public transportation BIT [6] is a project set forth by German Ministry of Education and Research. It focuses on the mobility support for moving vehicles and switching between various technologies. The core focus of the project is to support triple play like Streaming media and IPTV however we will discuss only on the logical architecture. BIT project provides a mobility solution for the fast moving vehicles. Commuters inside the train connect to the rail point server RPS that conducts various operations in order to establish train to land connectivity. While moving these vehicles constantly change their point of attachment POA to the land network. The POA connects the RPS to the rail point gateway RPG on the land. And then the 
RPG is responsible for providing fast and efficient Internet services. The RPS conducts following basic operations.

i . Authentication and Authorization AAA.

ii. Decision for new POA to another network.

iii. Rerouting of data packets.

iv. IP based mobility, using mobile IP protocol.

v. Transport protocols like stream control transport protocol SCTP.

vi. Changing IP addresses while maintaining ongoing connections.

The BIT based logical models is based on Mesh approach. Providing more than one transmission path minimizes reregistration times, packet delays and losses. Handovers taking place at data link layer DLL takes up to 2 seconds, causing bad QOS. The model proposes that the RPS holds several interfaces to various technologies and they can be simultaneously used to provide the desired QOS.

\subsubsection{Inter RBS Wireless Mesh based Topology}

Another proposal by the BIT project is to consider inter radio base station RBS mesh based connectivity, In order to avoid cost for laying cables between the RBS and faster communication through multiple paths for communication to the backbone. There is a need for a huge number of RBS along side of the railway track. To provide seamless handovers and IP-Mobility mesh based wireless connectivity between lands based RBS is a better solution. Figure 3.1.1 gives a pictorial representation of the logical architecture and Mesh based approach.

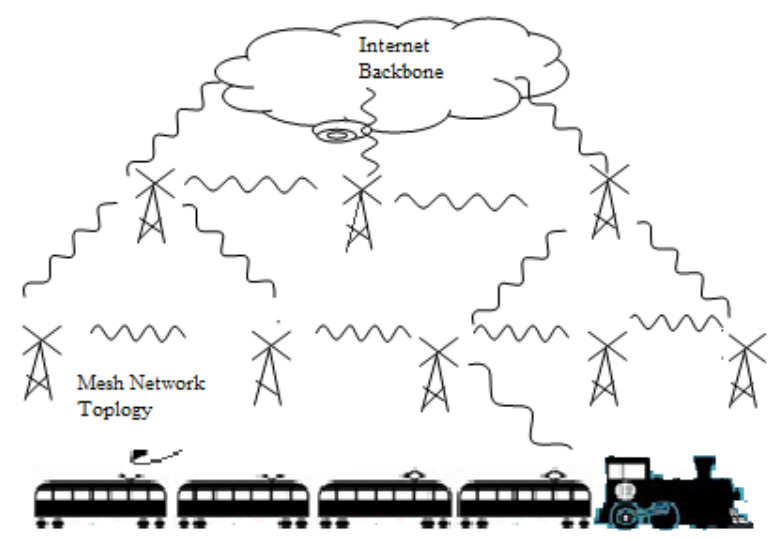

Figure 3.1.1 BIT Mesh Based Topology

\subsubsection{Novel Transport Layer NTP}

Transport layer is responsible for maintaining robust end-toend connectivity. Maintaining end-to-end connectivity is the core concept of improving QOS and in turn mobility support. The goal is to minimize the connection failures due to reasons like link failure or handover failure etc. It is observed that observed that most of the time end-to end connectivity failures are due to a single point of failure that might happen in a wired or wireless part of the network. If link failure is observed in the wired part routing protocols handle them efficiently by rerouting through an adjacent path. While in case of wireless connection failure it is normally due to errors in medium or Bit Error Rate BER, low bandwidth and interference. If the end-to-end connectivity is broken the overall service is ruined.

Therefore NTP provides a unique concept of multi-homed terminals. This technique involves more than one IP-Address assigned to a single wireless terminal and more than one routing path from end-to-end. Transport layer protocols TCP and UDP do not support multi-homing therefore it is proposed to use stream control transmission protocol SCTP on the transport layer.

\subsection{Tunnel Based QoS Management}

Tunnel based QOS management framework is a practically interesting solution for providing wireless Internet services to moving vehicles. The approach proposes satellite based connection to the vehicles like trains as unfit due to high delays in round trip propagation. The tunnel-based approach is set forth for technologies like Wi-Fi and WiMAX that provide high data rates on high-speed trains. It is the responsibility of aggregation network to transport data traffic between users and service providers by using tunnel-based approach. The approach provides details of tunnel set-up and tear down triggers in the Ethernet aggregation network for both Wi-Fi and WiMAX technologies. The main reason for choosing Ethernet level aggregation is because most of the operators make use of QOS-aware Ethernet switches. Ethernet networks use Spanning tree protocols STP, rapid STP and multiple STP for loop free active topology [7]. The overall QOS is improved using STP.

\subsubsection{FAMOUS Architecture}

Fast Moving Users architecture is proposed for cellular terrestrial based broadband service providers. It is based on the tunnel-based approach. WiMAX or Wi-Fi based radio base stations are installed alongside of the railway tracks, each base station is connected to the Aggregation Gate Way (AGW) that gives access to the aggregation network part.

The user's requests are aggregated into different groups based on traffic characteristics. The traffic of each aggregated group is multiplexed into the Virtual Local Area Network (VLAN) tunnel. The aggregation network is responsible for the transport of data traffic, by means of high bandwidth tunnels moving at high speed, to the service provider domain [9]. These connections between the service provider SP and aggregation network are realized as aggregation gateways AGW. Figure-3.2.1.a on next page shows the FAMOUS architecture.

At layer-2 Data Link Layer to achieve fully enabled Ethernet switched network, each device is configured with three features.

1. Virtual Local Area Network VLAN support.

2. IEEE standard 802.1 p-support providing two queues per mount.

3. GARP Wireless LAN Registration Protocol GVRP aware.

4. One of the Spanning Tree Protocol STP support.

Now while the train passes by the aggregation gateways AGW it establishes a physical link, so the train has multiple links with AGW in its way. But only one of the links is active at one time making a VLAN tunnel using spanning tree protocol STP. It is the functionality of the Control entity at the service gateway SGW to decide which tunnel to establish through which aggregation gateway AGW. As a result high speed, loop free path for data traffic is defined. resulting in best QoS. The scenario is depicted in Figure-3.2.1.b. on next page.

\subsubsection{Tunnel Establishment and Tear Down}

Only one tunnel needs to be established at one time while other in a disconnected state. The decision is made by the location information transmitted from the moving rail mounted base station to the aggregation gateway AGW. The train sends in location information in longitude-latitude using 
a GPS. On the basis of GPS information the service gateway SGW decides which tunnel to establish and others to tear down. The tunnel setup depends on the tunnel length however it takes $100 \mathrm{~ms}$ on average on one tunnel establishment.

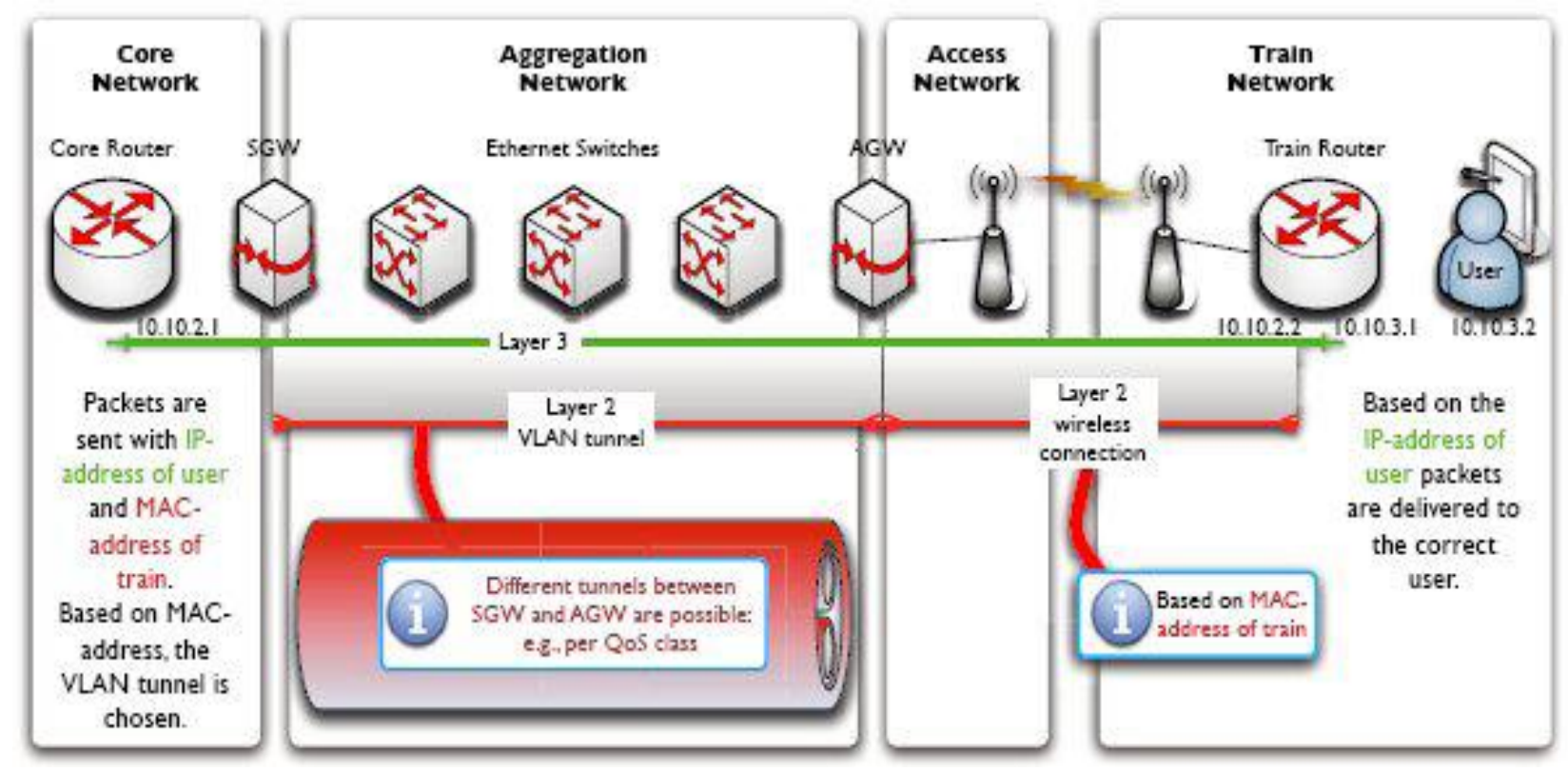

Figure 3.2.1.a Fast Moving User Architecture [10]

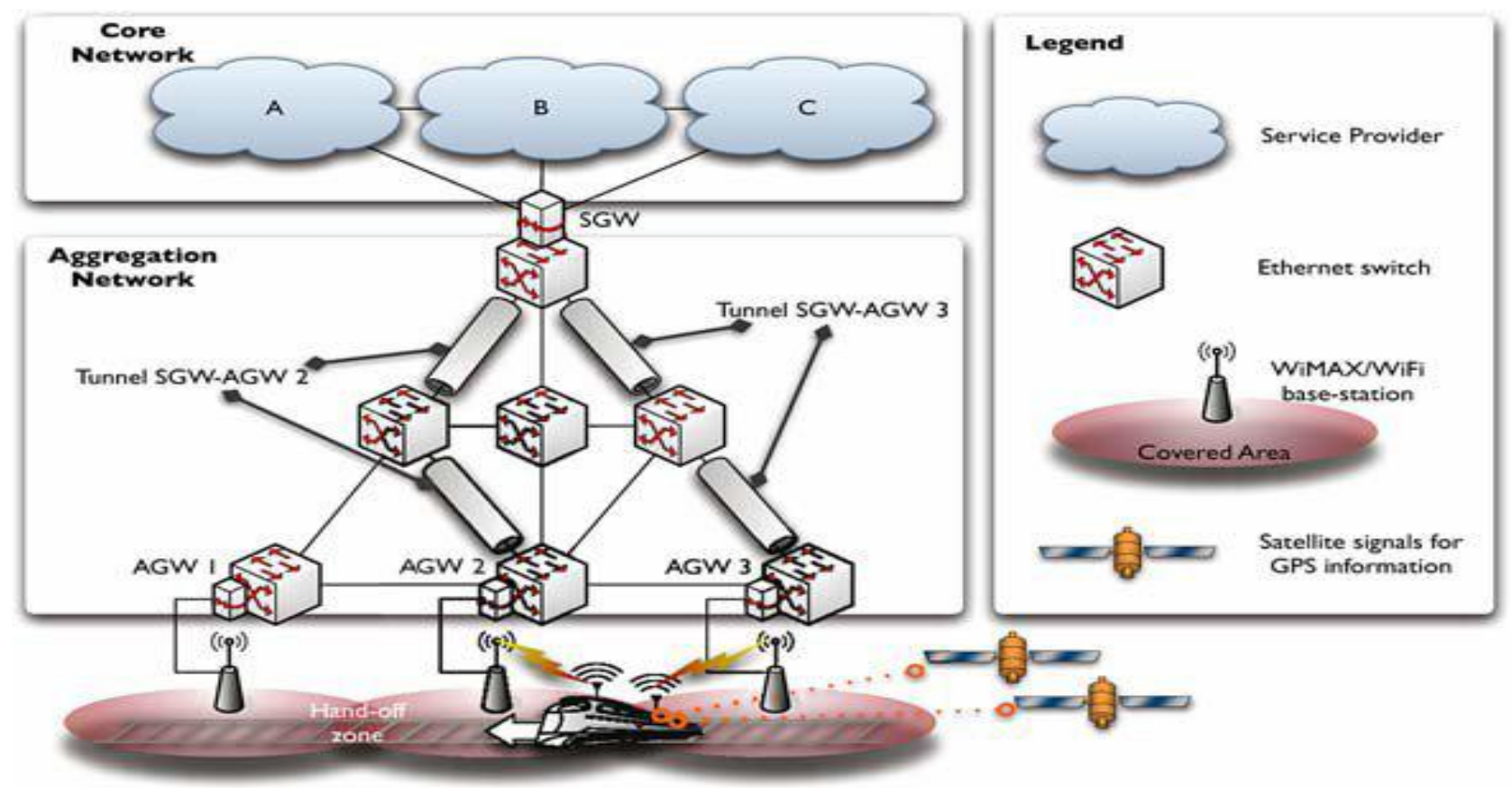

Figure 3.2.1.b Famous Architecture Tunnel Selection 


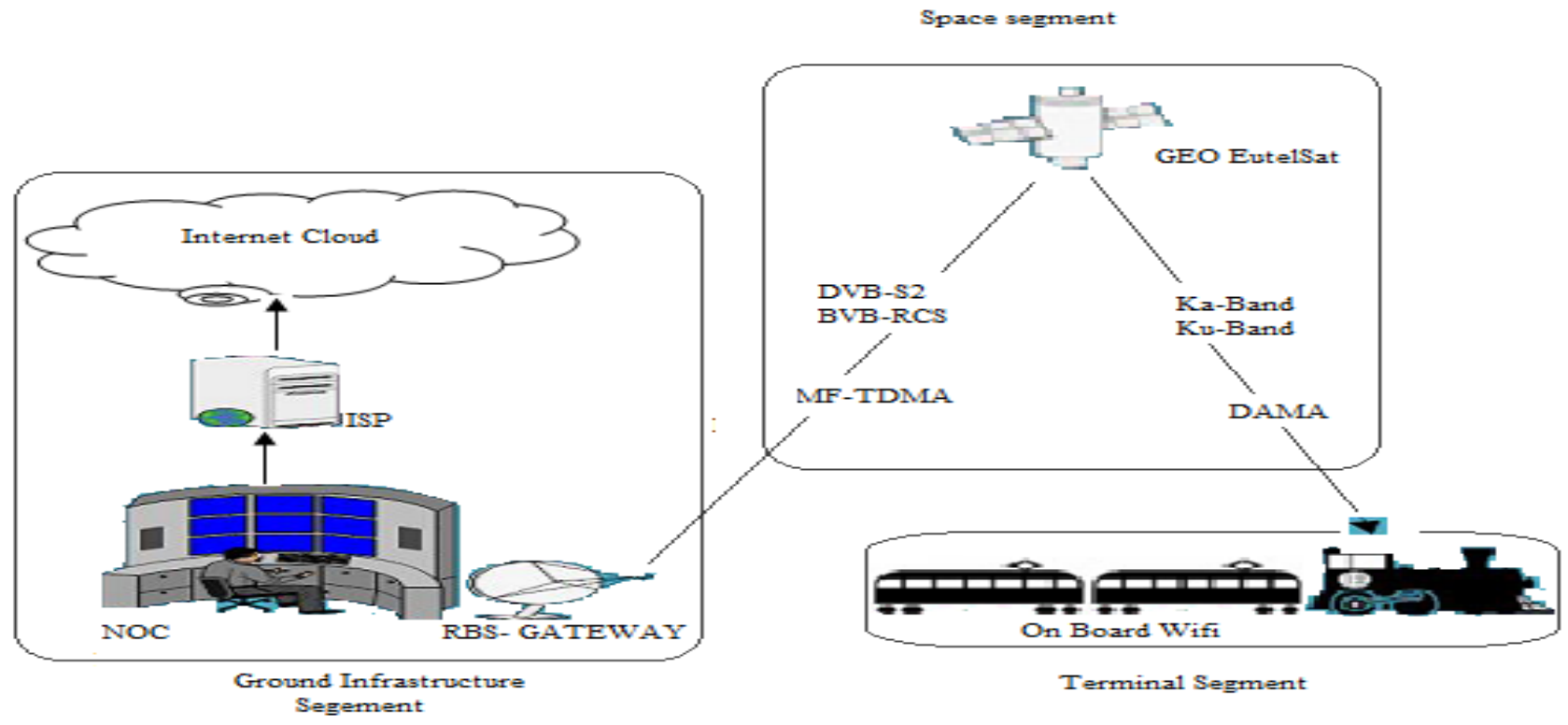

Figure 3.3 MOWGLY architecture and its segments

\subsection{MOWGLY Architecture}

MOWGLY 'Mobile Wideband Global Link System' is one of research projects undergoing at the platform of EU-FP6 projects. It is an architectural model, which provides details for implementing wireless broadband access on fast moving vehicles through satellite based connectivity. MOWGLY architecture proposes the use of Eutelsat GEO satellite constellation that is operating at $\mathrm{Ku}$ Band approximately 22000 Miles above earth equator. The adaptability can be improved using multiple spot-beam satellites at Ka Band. MOWGLY is based on DVB-S2 'Digital Video Broadcast Standard 2' and DVB-RCS 'Digital Video Broadcast reverse channel satellite' standards at the physical layer. MOWGLY architecture divides the network into three basic segments.

1. Ground Infrastructure segment consisting of NOC Network operation centre, routers and ISPs.

2. Space segment, consisting on bi-directional satellites and satellite arrays.

3. Terminal segment, consisting of fast moving vehicles like Train, Buss, Ship or an aero plane using broadband service onboard. MOWGLY architecture promises an efficient use of satellite bandwidth. The architecture will utilize the existing satellite infrastructure nothing new will be installed. On the reverse link the standardized multiple access schemes Demand

Assigned Multiple Access (DAMA) will be used, for an efficient, collision free utilization of network bandwidth [10]. On the downlink MOWGLY follows DVB-RCS standard. It is based on MF-TDMA 'Multiple Frequency Time Division Multiple Access', modulation schemes like Quadrature Phase Shift Keying (QPSK) and coding scheme like Turbo-Coding. A simplistic model of MOWGLY architecture is shown in the Figure-3.3

\subsection{Thin Client Based Architecture}

Thin client based architecture is a basic solution for mobile environment where clients have small, portable, insufficient battery time, limited processing capability and low storage devices such as mobile phone, PDA and MP3 Players and IMate. The architecture proposes a new approach to centralized processing. All the storage, processing, control and applications run at the remote centralized server and all thin clients act as a display module or input output interface. As a result storage is increased; faster processing and extended battery times are observed. In a fast moving train environment, thin client based architecture proves to be an ultimate solution. While the train is on the move the PoA changes each time when a handover to a new RBS occurs. Consequently application process is check pointed and the image is moved to a new application server causing delay in overall service and possible data loss. This application migration is a costly solution and time inefficient. Thin client based approach offers context aware access servers at the aggregation network level. The context awareness can be based on user behaviors like most of the users access emails, listen to music or view news pages. If context aware application servers are installed application migration can be reduced. It will be the responsibility of the aggregation network to compare and identify the context, which the user wants to access and connect the client directly to the application server or context provider. The thin client approach is based on user behavior and is very effective. For example train commuters in the morning time going to their offices, universities or businesses are most interested in their mails and news pages. They can be directly connected to the application provider above the context aware network and during handovers in the journey minimal handovers will be observed resulting in better QOS. It is a five-step model stated below [11].

1. Acquisition of context information from context providers.

2. Aggregation of context information, Knowledge Base.

3. Modeling of context information using ontology's, Context Model.

4. Reasoning and validation of context information, Context Reasoner.

5. Mapping context aware service to the context provider or Application Server. 
It is the functionality of aggregation management system AMS to acquire, aggregate and model context information on the basis of user behaviors. The user behaviors can be observed differently on the basis of specific time in a day, specific days in a week or over a longer period of time. The overall architecture provides support of better management services and low latency. Once the application server is connected to the thin client service migration is not needed any more for the mobility support. When a handover takes place only the aggregation gateway observes the change in PoA of a specific client. The control information is transferred from one aggregation gateway to another but the application server remains the same only PoA, and AGW is changed. Figure-3.4 gives a pictorial representation of the thin client architecture

\section{PRACTICAL DATA RATE OBSERVATION}

In order to observe the data rates offered on board, and experience Wi-Fi Internet access onboard, we chose GNER 'Great North Eastern Railway' route from London Kings Cross station till Peterborough (UK). GNER rails provide Wi$\mathrm{Fi}$ Internet service onboard using Icomera $\mathrm{AB}$ solution. Satellite based connectivity on uplink offers $2 \mathrm{Mbps}$ and $3 \mathrm{G} / 4 \mathrm{G}$ based connectivity of downlink provided by orange network offers $1 \mathrm{Mbps}$. This is the data rate promised by GNER for its customers at following rates.

- $\quad 30$ Minute use: $£ 2.95$

- 60 Minute use: $£ 4.95$

- 120 Minute use: $£ 7.95$

- 24 Hour use: $£ 9.95$

The journey time from London Kings Cross Station to Peterborough Station is 45 minutes. But the overall track rout is more than 12 hours long.

\subsection{Data Rate Monitoring}

For the network data rate analysis we chose Bandwidth Monitor [13], a freeware used to monitor network and Internet bandwidth. We observed the data rates on GNER Wi-Fi Internet on basis of three different scenarios.

In the first scenario we observer the offered data rates using normal browsing and chat applications. Normally commuters on train are more interested in E-mail, News, Chat and web browsing rather than using customized applications for
Audio/Video. Data rates offered by GNER were really appreciable and consistent, without any break or no network coverage points during the journey. Figure-4.1.a shows the graphical representations and averages of data rates.

Web browsing was tested rather than using customized applications for Audio/Video. Data rates offered by GNER were really appreciable and consistent, without any break or no network coverage points during the journey. Figure-4.1.a on next page shows the graphical representations and averages of data rates.

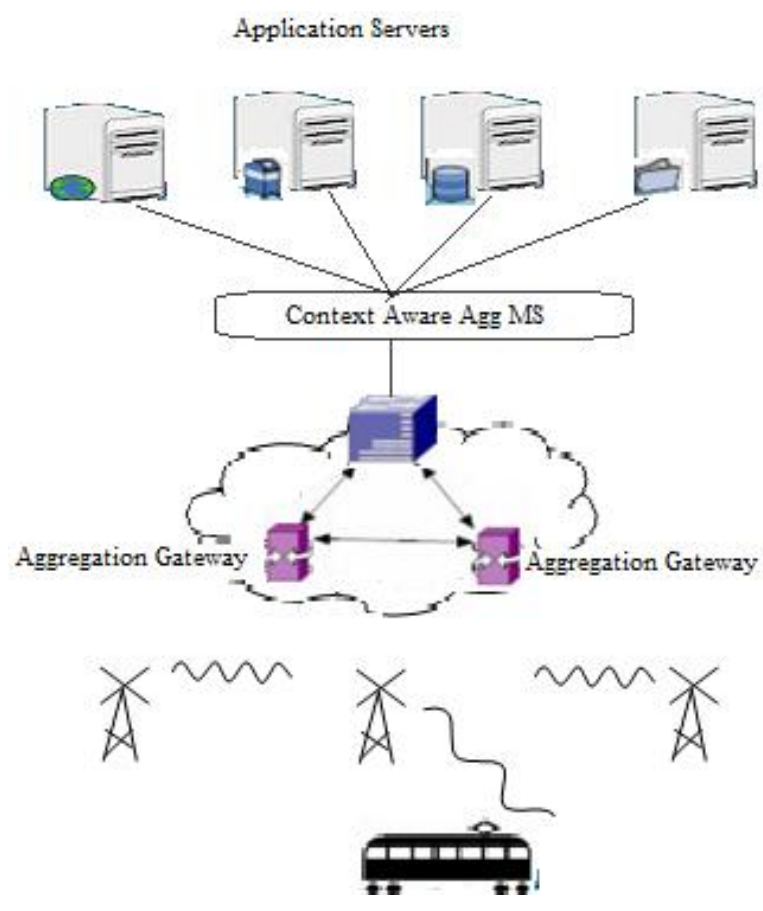

Figure 3.4 Thin Client Architecture 


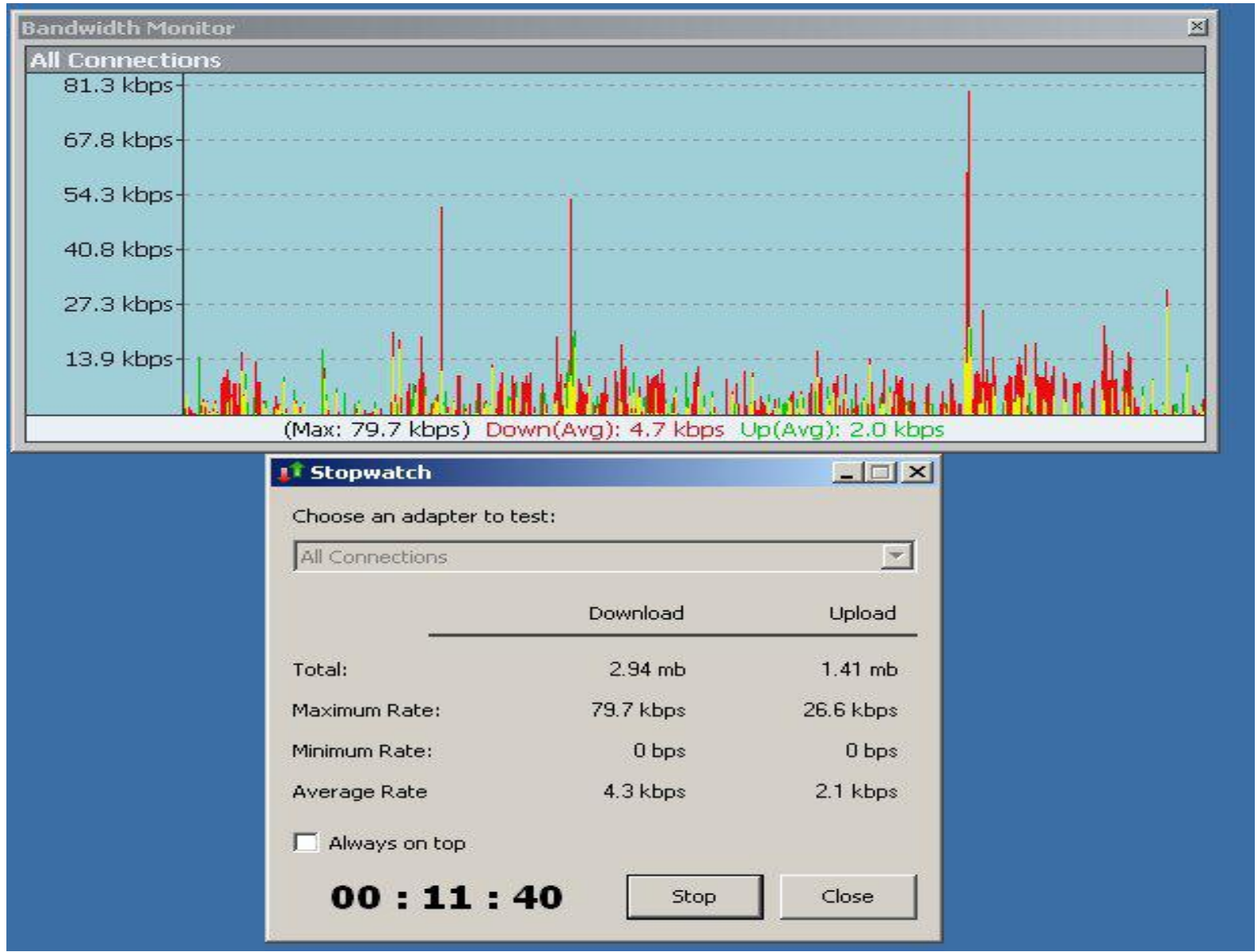

Figure 4.1.a Normal Browsing Scenario

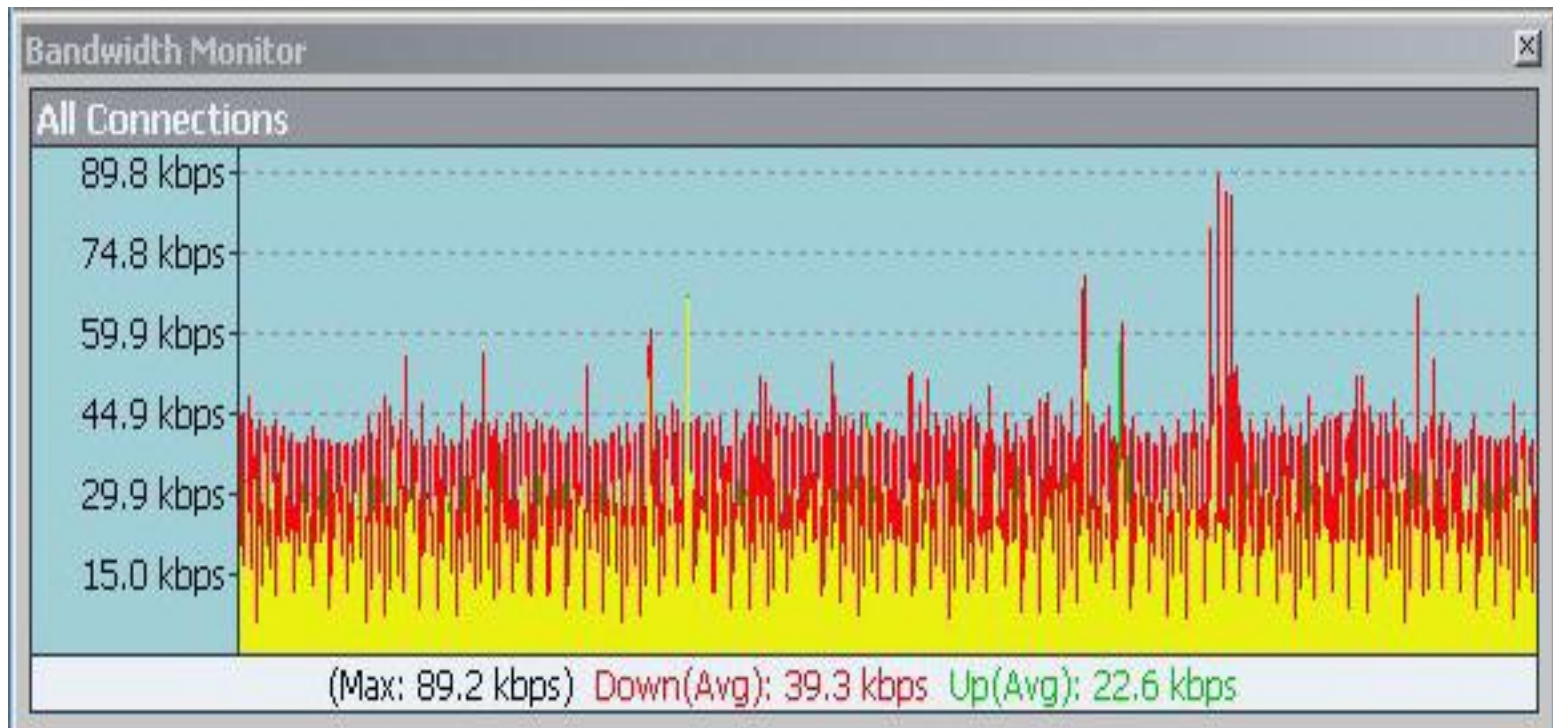

Fig 4.1b Data Rates for Max Downloads Radio cast 


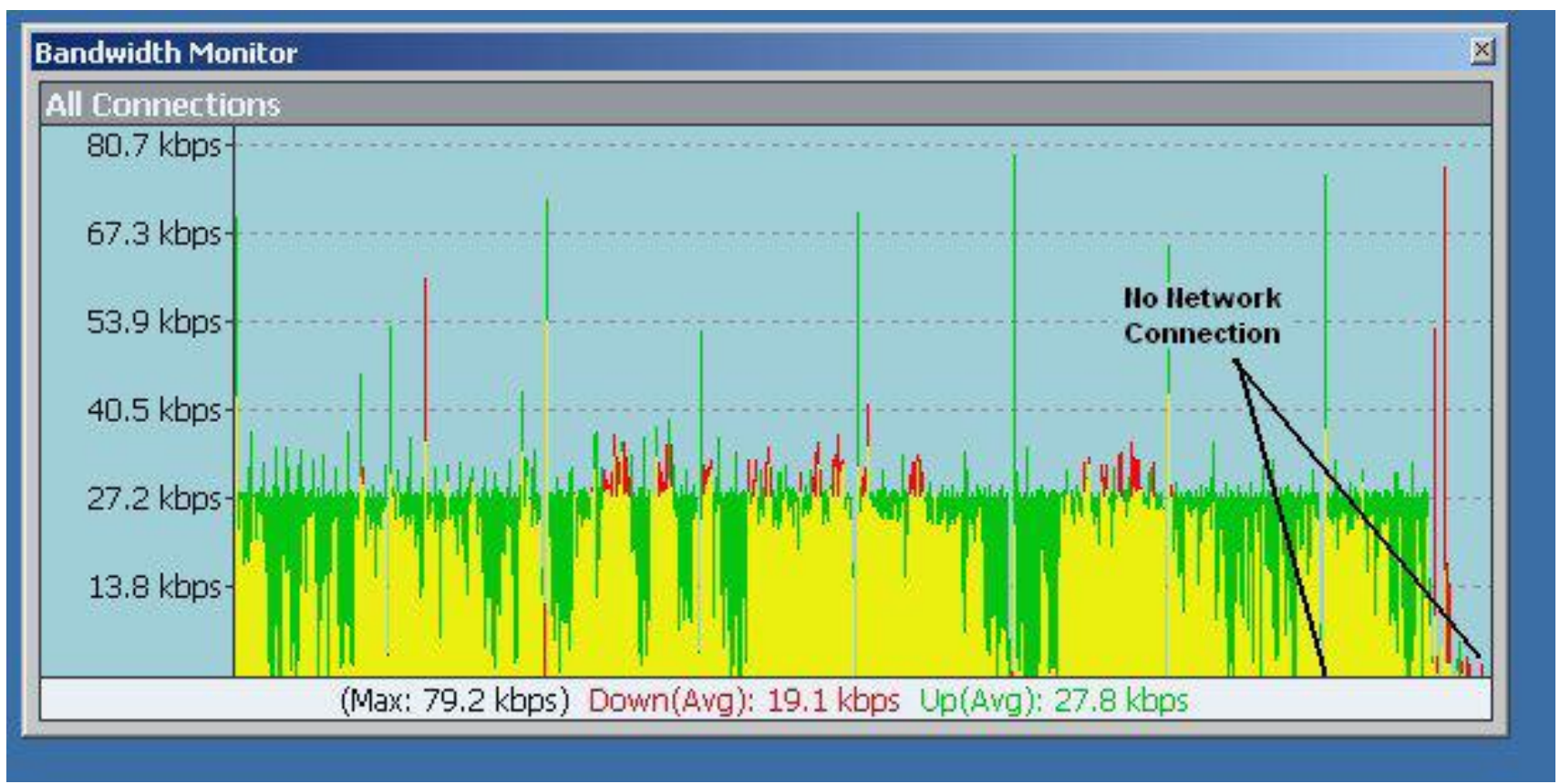

Figure-4.1.c Data Rates for Max Uploads in using VOIP service

In the second scenario we tried to observe the maximum download capacity of the GNER network. And see if it was possible to view live video streams using the Internet connectivity. Playing a video online took a long time and buffering was no consistently possible but live radio cast services could be heard properly without any long pauses and latency. This was really impressive. Commuter on train could listen to live radio using the Internet connection. Figure-4.1.b on previous page shows the average data rates and graph for live radio streaming.

In the third scenario the authors have decided to establish a voice call using VOIP 'Voice over IP' service on Wi-Fi Internet in GNER rail. The voice connection was established with a delayed in voice jittery voice but the overall meaning of a sentence could be understood. In a conversation of 3 minutes the VOIP connection broke down for 2 times and call establishment was difficult. But still it was possible to make a VOIP based phone call. Figure-5.1.c shows the data rates and graphical representation when the network was set to maximum stress on uploads. In all Figures, green color indicates upload, red color indicates download and yellow color indicates upload and download simultaneously. The overall service provided by GNER rail was appreciable but a bit expensive. A daily commuter on train might not be able to afford the prices. They need to develop a good market penetration plan. Since we could not find many people in first class lounge using the service offered. There were only two other passengers in the first class lounge excluding us, using Wi-Fi onboard Internet services. That is why we could see such good data rates at a high speed. If the number of passengers using Wi-
Fi service were more, the service quality would have degraded drastically. Apart from an inspiring Data

Rates for Internet offered on GNER rail we also observed following remarkable arrangements onboard.

- The offered data rates are acceptable if not up to the promised rates.

- Basic browsing and chat applications are carried out without any delays.

- Video and voice conferences are difficult to establish yet.

- GNER offers $100 \%$ connectivity, and it is observed that the connectivity was without any network disconnections apart from one or two points during the journey.

- $\quad$ GNER has ensured Wireless signals are not hurdled while passing through the tunnels and provide consistent availability

- Onboard staff is very helpful in assisting you to connect the wireless facility.

- Onboard safety is ensured using Wireless CCTV live recording.

- Train times and weather updates are reported actively.

- $\quad$ The Wi-Fi onboard coverage is provided throughout the rail network at every destination route.

- Power outlets are available on each seat as in case of longer journey the battery charging is required.

The basic steps involved in establishing a Wi-Fi connection onboard were given on a broacher. These steps are very easy to follow and start enjoying the Internet facility onboard. The overall activity is summarized in Table-4.1.d 
Table 4.1.d Overall Data Rate Analysis

\begin{tabular}{|c|l|l|l|}
\hline & $\begin{array}{l}\text { Test } \\
\text { case-1 } \\
\text { Basic } \\
\text { Browsing }\end{array}$ & $\begin{array}{l}\text { Test } \\
\text { Case-2 } \\
\text { Live } \\
\text { Radio } \\
\text { (Voice) }\end{array}$ & $\begin{array}{c}\text { Test Case-3 } \\
\text { VOIP Services \& } \\
\text { Video Streaming }\end{array}$ \\
\hline $\begin{array}{c}\text { Average } \\
\text { Upload }\end{array}$ & $2.0 \mathrm{Kbps}$ & $22.6 \mathrm{Kbps}$ & $27.8 \mathrm{Kbps}$ \\
\hline $\begin{array}{c}\text { Average } \\
\text { Download }\end{array}$ & $4.0 \mathrm{Kbps}$ & $39.3 \mathrm{Kbps}$ & $19.1 \mathrm{Kbps}$ \\
\hline $\begin{array}{c}\text { Maximum } \\
\text { Data Rate }\end{array}$ & $79.7 \mathrm{Kbps}$ & $89.2 \mathrm{Kbps}$ & $79.2 \mathrm{Kbps}$ \\
\hline $\begin{array}{c}\text { Minimum } \\
\text { Data Rate }\end{array}$ & $0.0 \mathrm{Kbps}$ & $0.0 \mathrm{Kbps}$ & $0.0 \mathrm{Kbps}$ \\
\hline $\begin{array}{c}\text { Point of No } \\
\text { Converge }\end{array}$ & No & None & 2 \\
\hline $\begin{array}{c}\text { Time to } \\
\text { Application } \\
\text { Action }\end{array}$ & $<2 \mathrm{Sec}$ & $<50 \mathrm{Sec}$ & $<3 \mathrm{Minutes}$ \\
\hline $\begin{array}{c}\text { Customer } \\
\text { Satisfaction }\end{array}$ & Yes & Yes & No \\
\hline $\begin{array}{c}\text { Quality of } \\
\text { Experience }\end{array}$ & Excellent & Good & Bad \\
\hline \multicolumn{2}{|c|}{} & & \\
\hline
\end{tabular}

\section{TURNING NEED FOR Wi-Fi ON TRAIN INTO DEMAND}

Before implementing any technological venture business plans are made, market surveys are conducted, customer trends are visualized and on the basis of target market characteristics a business is said to be profitable. Wi-Fi Internet access on trains was perceived to be one of the technological breakthroughs but it has not gained much popularity in terms of customer base yet

According to business plan proposed to CCIT 'Capital Corridor Intercity Train' BWCS 'Business Wiring Consultants' conducted a survey in 1600 UK train commuters. Train passengers are expected to spend $\$ 420$ Million per year on Wi-Fi services onboard [12].

- $78 \%$ of Business travelers are interested in using Wi-Fi on trains.

- $72 \%$ would prefer to use trains instead of plains if Wi-Fi services are available.

- $90 \%$ users are willing to pay $10 \$, £ 4,60$ SEK provided a good QOE 'Quality of Experience' is guaranteed.

- $\quad 44 \%$ Business Class customers showed willingness to pay for the service while $56 \%$ wanted it for free.

- $\quad 80 \%$ customers consider Wi-Fi on train useful only if traveling time is more than 1 hour.

- GNER, UK has calculated that $22 \%$ of journeys have shifted from Air to Rail due to offering of WiFi on trains [13].

The statistics collected by various surveys show that the target market currently is focused only on business travelers and 1st Class customers. And amongst them only 50\% are expected to carry a laptop or PDA onboard. Therefore the current target market is very small as compared to overall mass level of passengers on train. The question is how to make this service more demanded and turning it into an irreplaceable accessory while travelling on a train. Certain strategies can be adopted to make the Wi-Fi services more attractive for potential customers.

\subsection{Introducing VAS Value Added Services}

The basic functionality of Wi-Fi onboard is to provide wireless Internet access to the train passengers. To make the onboard experience more meaningful, comfortable and enjoyable many value added services could be offered along with the basic Internet service. Most of the VAS 'Value added services' proposed are within the train environment there will be no need for the RPG 'Rail Point Gateway ' to utilize the AGW 'Aggregation network' bandwidth for providing these services only a web portal can be designed with an attractive GUI. This portal can contain links to all the VAS onboard. These services can be offered making optimal use of Wi-Fi IEEE $802.11 \mathrm{a} / \mathrm{b} / \mathrm{g}$ technology already available onboard without any external network connection. Some examples of value added services are given below.

i. AAA 'Accounting Authentication and Authorization' server at Rail point server (RPS) can be programmed to offers Usage Based Billing instead of hourly usage. As a result a customer will be billed according to bytes of data uploaded and downloaded.

ii. Introducing a Multimedia Server onboard, containing a database of onboard movies and music videos. As a result the same Wi-Fi service onboard will be used for multimedia content provider.

iii. Using the same Multimedia Server onboard we can offer updated electronic newspapers, journals and magazines on daily basis.

iv. Introducing a Game Server onboard offering single user and network-based games turning onboard experience into a gaming Zone.

v. Almost every train service is equipped with a GPS 'Global Positioning Service' antenna. Using the features on GPS and Wi-Fi environment on train the service provider can offer Location Based Services. For example if a passenger needs to hire a taxi at next station he might be able to book a taxi online. Similarly room booking in a hotel, famous holiday resorts and visiting places around a particular station etc.

vi. As in Aero planes Tax Free shopping is available. Onboard Low-priced E-Stores can be introduced. If a train commuter wants to buy a gift item or a gadget onboard he just needs to place an order looking at the product using Wi-Fi network web portal and the item can be delivered on his seat.

vii. Normally most of the train services offer cafeteria services onboard but the customer needs to leave his seat in order to buy something. By introduction of $\mathrm{Wi}-\mathrm{Fi}$ based Online Cafeteria Onboard customer comfort ability onboard can be confirmed.

viii. Customers do not carry liquid money inform of cash these days they prefer credit cards and debit cards rather than currency notes. Onboard Credit Card billing can attract a mass level on customers. It just needs secure connection implementations. As a result tickets can be bought on train using a credit card. An external connection from train to land will be required for this service.

ix. CCTV Onboard can provide security and surveillance making the overall journey more reliable and secure.

$x$. Train Staff Communication with each other using the same Wi-Fi technology using PDA or customized hardware. Even voice or Video communication between train staff can be made possible using Wi-Fi technology. This approach makes easier and faster trouble notification and handling onboard. 
xi. TV Broadcasting can be achieved on a train using DVB satellite link directly for video broadcasting purposes.

\subsection{Improving Quality of Experience}

Every broadband user on train is also using Internet services in their offices and homes. They expect the service to be as fast, consistent and efficient as they experience it at other places. Despite of the fact that trains are at high speed the customer's expectation has to be achieved. Providing Internet on trains is a high CAPEX and OPEX venture but by introducing just small changes in architecture, pricing and technology the overall service perception in customers mind can be improved a lot and market demand for Wi-Fi onboard can be increased.

i. Adopting 'Thin client based architecture' can improve the overall data rates and quality of service drastically. It is more like a client server environment where the requests are sent to the server and all the processing is done at the server end as a result overall network traffic is reduced.

ii. Making use of routing protocols to make shortest roundtrip times, using standardized logical architecture depending on needs. For example, MOWGLY's famous architecture.

iii. Replacing backend network Wireless links with Optic Fiber based links, offering minimum data loss and fast data transfer.

iv. Always prefer Mesh topology so that if one of the links in down, the other has an alternate route for data transfer and service.

v. Reducing and removing no coverage points such as tunnels, mountains, deserts and uneven landscapes by introducing Hybrid Technology Solutions.

vi. Use Multiple Links of same technology at one time providing, higher accumulative data rates and failure prevention in form of Active Standby.

\subsection{Market Penetration Plans}

The Wi-Fi Internet onboard technology is already commercialized. Now it needs effective marketing plans for gaining customer attention and acceptance. Market surveys conducted by various organizations clearly indicate that people are interested for the service and are ready to pay for but still customer base in terms of service users is very small. Some strategies for rapid market penetration are stated below. i. Changing target market focus form Business and First Class passenger to every commuter on train. Making train environment more spacious, comfortable and tempting for every user to bring along his/her PDA or Laptop when they are going on a journey, rather than sitting idle and looking out of the window.

iii. Increasing customer awareness by various advertising activities such as Broachers, Radio, and Television and NEWS advertisements. Making them realize the fact that they are wasting their precious time, which they can utilize more productively.

iv. Introducing multiple pricing plans on the basis of Utilization, Total time, Occupation, Age, Monthly and weekly packages.

v. Clear out the pricing instead of disguising it in other charges.

vi. Providing onboard Internet surfing facility for free as an introductory offer, for a few days after service launch.

vii. Introducing corporate level packages, providing Internet services to corporate employees on both pre-paid and postpaid packages.

viii. Introducing incentives for the customers on train using Wi-Fi service, such as lottery nominations or free drinks etc. ix. Making the journey on trains more of a joy ride with Wi-Fi availability.

Implementing such changes in terms of VAS, Enhancements of data rates and Market penetration plans Wi-Fi Internet access onboard will become a market breakthrough much earlier than anticipated.

\section{CONCLUSION}

Wi-Fi Internet access on trains is relatively new but fast growing technology. Almost every railroad company is has either adopted this technology or they are looking forward to. Many standard and non-standard solutions are proposed and implemented by wireless solution providers across the world, each having its own pros and cons. Due to the efforts of these vendors and operators $\mathrm{Wi}-\mathrm{Fi}$ broadband internet access onboard is possible today but still it is not a market breakthrough. There are many reasons to this problem, which are discussed in previous chapters. The commercialization of Wi-Fi on trains and other fast moving vehicles is a giant leap as far as technology is concerned. But there is a need for aggressive advertising, effective marketing plans and continuous improvements in the services provided to make this technology successful in terms of generating revenues for the operators.

\section{REFERENCES}

[1] Shigetoshi Kudoh, "Wireless Broadband in Japan", September-12, 2006 Tekes, Finnish Funding Agency for Technology and Innovation. http://www.tekes.fi/

[2] "High speed WiMAX Connectivity on rail line". August-1, 2006 News Report. URL: http://www.govtech.net/digitalcommunities/story.p $\mathrm{hp}$ ?id=100383

[3] Bart Lannoo, Didier Colle, Mario Pickavet, and Piet Demeester, Ghent University "Radio-over-FiberBased Solution to Provide Broadband Internet Access to Train Passengers"

[4] X. Liang, Chan and R.E. Sheriff, "Mobile Internet Access for High-speed Trains via Heterogeneous Networks". Communication systems research group, University of Bradford. Pages 180-181.

[5] Huber + Suhner AG. "Huber and Suhner Antennas Railway Antenna Application" Mobile Communications + Electronics Sector Communication Equipment Components CH-9100 Herisau , Switzerland. Pages 1-3. URL: http:// www.hubersuhner.com

[6] Andreas Roos, Nico Bayer, Dmitry Sivchenko, Peyman Behbahani, icheal Flegl, Dr. Gerard Kadel. "Broadband Wireless Internet Access in Public Transport". International Research Project by German Ministry of Education and Research. Pages 2-3.

[7] IEEE 802.1. Standards for local and metropolitan area networks. 
[8] Frederic Quickenborne, Filip de Greve, Filip de Turck, Ingrid Moerman and Piet Deemester. "A Tunnel Based QOS Framework for Delivering Broadband Internet On Trains". Department of IT, Ghent University - IBBT. Pages 2-7.

[9] P. Vincent, A. Arcidiacono. Chevet, L. Audounet, G. Naym, Alvarez, L. Babarit. "Mobile Wideband Global Link sYstem' (MOWGLY) Aeronautical, Train and Maritime Global High-Speed Satellite Services". Pages 3-5.

[10] M. Strobbe, P. Simoens, L. Deboosere, D. De Winter, F. Van Quickenborne, F. De Greve, F. De Turck, P. Demeester. " Support for Moving Users through Thin Clients : Hype or Future?" IBCNINTEC,
[11] Bandwidth Monitor "Monitor Network and Internet Bandwidth"

URL: http://www.bwmonitor.com/

[12] Hamed Benouar CCIT Director, Adib Kanafani, Jim Alliason, Bensen Chiou, Harsh Verma, Adam Dankberg, Ipsita Banerji. November 2007 "California Trains Connected Business Model Research” Pages 16,19, 26-28

[13] Business news on ZDNet website "GNER onboard Wi-Fi arrives early". July 2006. URL: http://news.zdnet.co.uk/communications/0,1000000 085,39279979, 